\title{
An Efficient CRT based Digital Image Watermarking using Double Density Wavelet Transform
}

\author{
Prerna Singh \\ Ambedkar Institute of Advanced Communication Technologies and Research \\ Govt. of NCT of Delhi, India
}

\begin{abstract}
Digital Watermarking is an unpretentious and effective approach to afford copyright fortification. Watermark transparency is obligatory primarily for copyright protection. The challenge is to introduce a digital watermark that is both transparent and highly robust to common signal processing and possible attacks. The two basic requirements for an effective watermarking scheme, robustness and transparency, conflict with each other. We propose a watermarking technique for digital gray scale images that is based on utilizing congruence's in number theory and its generalizations in abstract algebra which have been developed in the context of image compression. Our watermarking scheme is based on a Chinese Remainder Theorem along with Double Density Discrete Wavelet Transform framework individually which allows for the possibility of directly watermarking the Image bit-stream, Watermark region selection is based on transformation level. In proposed scheme, initially, the cover image and watermark are transformed into spatial domain using Double Density Wavelet Transform and then singular values of these transformed images are combined using CRT coefficients. Our scheme is shown to provide very good results both in terms of image transparency and robustness. An optimal watermark embedding method is developed to achieve minimum watermarking distortion. Experimental results are provided in terms of Peak signal to noise ratio (PSNR), Mean Squared Error (MSE), Correlation and Weighted Peak signal to noise ratio (WPSNR) to demonstrate the effectiveness of the proposed algorithm.
\end{abstract}

\section{Introduction}

Digital media offers several distinct advantages over analog media, such as high quality, easy editing high performance and easy duplication. The high spreading of broadband networks and new developments in digital technology has made ownership protection and authentication of digital multimedia a very important issue. Digital watermarking provides a possible solution to the problem of easy editing and duplication of images, since it makes possible to identify the author of an image by embedding secret information in it. Digital watermarking technique is one of the solutions to avoid unauthorized copying or tampering of multimedia data. Recently many watermarking schemes have been proposed to address this problem. Digital Watermarking is defined as the process of hiding a piece of digital data in the cover data which is to be protected and extracted later for ownership verification [1]. Some of the important applications of watermarking technique are copyright protection, ownership verification, finger printing, and broadcast monitoring. The features of watermarking include robustness and perceptibility. Robustness indicates the resistivity of watermark against different types of attacks such as cropping, rotating, scaling, low pass filtering, resizing, and addition of noise, JPEG compression, sharpness, histogram equalization and contrast adjustment. Those attacks are either intentional or unintentional. It is well known that there are three main mutually conflicting properties of information hiding schemes: capacity, robustness and indefectibility [2]. It can be expected that there is no a single watermarking method or algorithm with the best quality in the sense that three mentioned above properties have the maximum value at once. But at the same time it is obvious that one can reach quite acceptable quality by means of combining various watermarking algorithms and by means of manipulations in the best way operations both in the spatial and in the frequency domains of an image. In paper [3], an approach to combining of DWT and DCT to improve the performance of the watermarking algorithms, which are based solely on the DWT, is proposed. The combination of these two transforms improved the watermarking performance considerably when compared to the DWT-only watermarking approach. As a result this approach is at the same time resistant against copy attack. In addition, the fragile information is inserted in a way which preserves robustness and reliability of the robust part.

Robustness is the property which is important for ownership verification whereas the fragility is important for image authentication. Robustness of watermarking algorithm is obtained to a maximum level when information is hidden in robust 
components of cover data. The increasing perceptibility will also decrease the quality of watermarked image. Generally information could be hidden, directly by modifying the intensity value or pixel value of an image or its frequency components [4]. The former technique is called spatial domain technique and later is called frequency domain technique. To obtain frequency components of an image, it needs to be transformed using any one of the transformation techniques such as Discrete Fourier Transformation (DFT), Discrete Short Fourier Transformation (DSFT), Discrete Cosine Transformation (DCT) [5], [6], Walsh Hadamard Transformation (DHT) [7], [8], and Discrete

Wavelet Transformation (DWT) [9], [10], [11], [12]. In transform domain, casting of watermark can be done in full frequency band of an image or in specific frequency band such as in low frequency band or in high frequency band or in middle frequency band.

Chinese Remainder Theorem (CRT) has been widely used as a solution to the problem of converting a number to a residue number system using general moduli set [13]. Recently CRT has been applied for several engineering applications, e.g., in dynamic access control in computing systems [14], encoding in communication systems in cryptography [15], and authentication in sharing of secrets [16], [17]. Recently Patra et al. have reported few watermarking techniques based on CRT [18] [20. It is shown that the CRT-based spatial domain watermarking technique is highly computationally efficient compared to SVD-based scheme and able to withstand several common attacks, but it cannot withstand JPEG compression [18]. However, in subsequent reports it is shown that the CRT based watermarking scheme implemented in DCT domain is able to withstand JPEG compression at the expense of a little more computational cost [19], [20].

This paper develops a hybrid digital image watermarking algorithm which satisfies both imperceptibility and robustness requirements. In order to achieve objectives this research used singular values of Transformation's (double density wavelet transform) sub bands to embed watermark. Further to increase and control the strength of the watermark, this work used an efficient CRT model. First both the cover image and watermark are transformed through Double Density Discrete Wavelet Transform, and then the proposed model calculated the singular values of transformed part of both cover image and watermark image using singular value decomposition. After that, the singular values are combined with each other by taking CRT factor between cover image and watermark image in order to evolve a more promising approach in field of digital image watermarking. The watermarking schemes proposed here are combined DD-DWT based processes, where the benefits of DWT are taken into consideration in choosing the most proper sub-band for watermark embedding in order to provide both robustness and imperceptibility and hence the LL sub-band is chosen after performing one level DD-DWT on the host image. Secondly, SVD is applied on the DWT sub-bands and for watermark embedding purpose the singular values are selected to provide further robustness to the schemes.

The association steps of this paper are as follows. The Introductory Section ends with a brief introduction of digital watermarking and its necessity in copyright protection. The introduction shows a brief explanation about digital watermarking, techniques for image watermarking and requirements for watermarking algorithms in brief.

Section 2 explains a General review and related work of digital image watermarking, many techniques have been proposed for the digital watermarking which are categorized in this section.

Section 3 addresses the proposed methodology and system model along with the technical specifications of proposed work including the steps of work and block diagram of both the embedding and extraction process.

Section 4 gives details about the simulation results; it also shows some comparative graphs which prove that the proposed approach overcome the traditional DWT-CRT based approach. Section 5 shows the observations, discussion and a general conclusion of the paper is presented.

\section{Proposed Method}

By taking up the design of discrete wavelet transforms based on oversampled filter banks. In this case, the wavelets form an over complete basis, or frame. In particular, we consider the design of systems that are analogous to orthonormal wavelets that is, the design of minimal length wavelet filters satisfying certain polynomial properties, but now in the oversampled case. The proposed wavelets are constructed using maximally flat FIR filters in conjunction with extension methods for paraunitary matrices. Because there are more degrees of freedom in the design problem, the wavelets described in this chapter are much smoother than orthonormal wavelets of the same support.

To develop the double density DWT we begin by selecting an appropriate filter bank structure. The filter bank illustrated in Fig. 1 exactly matches the strategy for sampling the time-frequency plane illustrated in the second panel.

This resembles the usual two-channel filter bank used in implementing the critically sampled DWT; however, the down-sampler and up-sampler in the high-pass channel have been deleted. This is called an oversampled filter bank because the total rate of 
the sub-band signals $\mathrm{c}(\mathrm{n}), \mathrm{d}(\mathrm{n})$ is exceeds the input rate by $3 / 2$. The double density DWT is then implemented by recursively applying this filter bank on the low-pass sub-band signal $\mathrm{c}(n)$. The prominent issue is the design of the filters $\mathrm{h}_{0}(n)$ and $\mathrm{h}_{1}(n)$ so that $\mathrm{y}(n)=\mathrm{x}(n)$. To construct a double density DWT with FIR filters we will use the oversampled filter bank shown in Figure 1. The filter $\mathrm{h}_{0}(n)$ will be a low-pass (scaling) filter, while $\mathrm{h}_{1}(n)$ and $\mathrm{h}_{2}(n)$ will both be high-pass (wavelet) filters. To develop the perfect reconstruction conditions we use standard multirate identities to write $\mathrm{Y}(z)$ in terms of $\mathrm{X}(z)$.

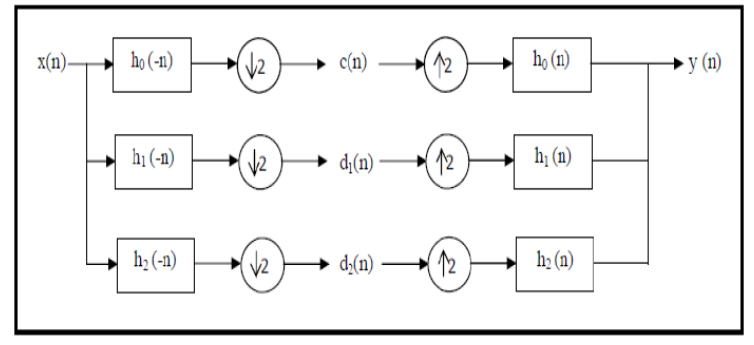

Figure 1. An oversampled analysis and synthesis filter bank permitting perfect reconstruction with FIR filters

\subsection{Double Density Discrete Wavelet Transform (DD-DWT) and Singular Value Decomposition (SVD)}

DD-DWT decomposes images into four bands. In this work, the watermark is embedded in maximum energy bands (means all frequencies). And after that, all bands are further transformed using singular values, this result in robustness to a wide range of attacks. SVD is an optimal matrix decomposition technique. It packs maximum energy into as few coefficients as possible. SVD has the ability of adapting to variations in local statistics of an image. So, watermarking schemes using SVD are typically of large capacity. Different types of wavelets are also used in experiment including 'db1' or Haar Wavelet, 'db2' or Daubechies level two wavelets, 'db4' or Daubechies level four wavelets, 'sym2' or Symlets level two wavelets, 'dmey' or Discrete Meyer wavelets and ' $\mathrm{db} 45$ ' or Daubechies level 45 wavelets.

\subsection{Overview of Singular Value Decomposition}

Singular value decomposition is a linear algebra technique used to solve many mathematical problems [29]. The theoretical background of SVD technique in image processing applications to be noticed is [30]:

a) The SVs (Singular Values) of an image has very good stability, which means that when a small value is added to an image, this does not affect the quality with great variation.

b) SVD is able to efficiently represent the intrinsic algebraic properties of an image, where singular values correspond to the brightness of the image and singular vectors reflect geometry characteristics of the image.

c) An image matrix has many small singular values compared with the first singular value. Even ignoring these small singular values in the reconstruction of the image does not affect the quality of the reconstructed image.

\subsection{A CRT coefficients model for double density wavelet transform domain image Watermarking}

A relationship between a DD-DWT domain human visual model and the modification threshold of singular values is established. The threshold, which in image adaptive, is used to determine the watermarking strength and guarantees the imperceptibility of the watermark.

Chinese Remainder Theorem (CRT): The model that is given below is used for the calculation of spatial domain profile for a given image. The Chinese Remainder Theorem is briefly given below.

\subsection{CRT coefficients calculation}

Let $\{m, n\}$ denote a pair-wise co-prime positive integers. The dynamic range, $N$ is given by $N=m . n$. According to CRT, for any given pair of positive integer, $\{p, q\}$, where $p<m$ and $q<n$, there exists a unique integer $Z$, such that $Z<N$. Calculation of $Z$ is as follows. First determine $r l$ and $r 2$ as:

$$
\begin{aligned}
& r_{1}=N / m=n \\
& r_{2}=N / n=m
\end{aligned}
$$

Next, find $s_{1}$ and $s_{2}$ such that (2) is satisfied:

$$
\begin{aligned}
& \left(r_{1} s_{1}\right) \bmod m=1 \\
& \left(r_{2} s_{2}\right) \bmod n=1
\end{aligned}
$$

Then, we find the unique integer $\mathrm{Z}$ as:

$$
Z=\left(p . r_{1} s_{1}+q . r_{2} s_{2}\right) \bmod N
$$

\subsection{The Inverse CRT}

Using inverse CRT an integer $Z, 0 \leq Z \leq N-1$, can be represented by a unique pair of integers, $\{p, q\}$, where $p<m$ and $q<n$. The values of $p$ and $q$ are determined as:

$$
\begin{aligned}
& p=Z \bmod m \\
& q=Z \bmod n
\end{aligned}
$$


The following variables are used in the proposed algorithm.

$$
\begin{array}{r}
D=\max \{m, n\}-1(5) \\
d=|p-q|(6) \\
b=p+q(7)
\end{array}
$$

\section{Watermark Embedding}

The algorithm for Watermark Embedding is represented in the form of block diagram as shown in Figure 2.

Step 1: For a given $\mathrm{N} \times \mathrm{N}$ cover image, apply the double density discrete wavelet Transform up to 3rd level, which produce a total of 9 bands wavelet coefficients.

Step 2: The low frequency sub-bands LL from 2nd level of wavelet decomposition are used to get 3rd level decomposition.

Step 3: Apply singular value decomposition on the DD-DWT transformed higher energy sub-band of image in order to calculate singular values.

Step 4: Similar operation performed on the watermark. After calculating singular values of both the cover image and watermark, say $\mathrm{S} 1$ and $\mathrm{S} 2$. We have to combine them using perceptual factor of CRT as:

$$
\mathrm{S}=\mathrm{S}_{1}+f_{c r t} \mathrm{~S}_{2}
$$
DWT.

Here, $f_{\text {crt }}$ is the CRT value calculated as per

Step 5: Then singular value $\mathrm{S}$ is again combined with the unitary matrix of cover image in order to restore it.

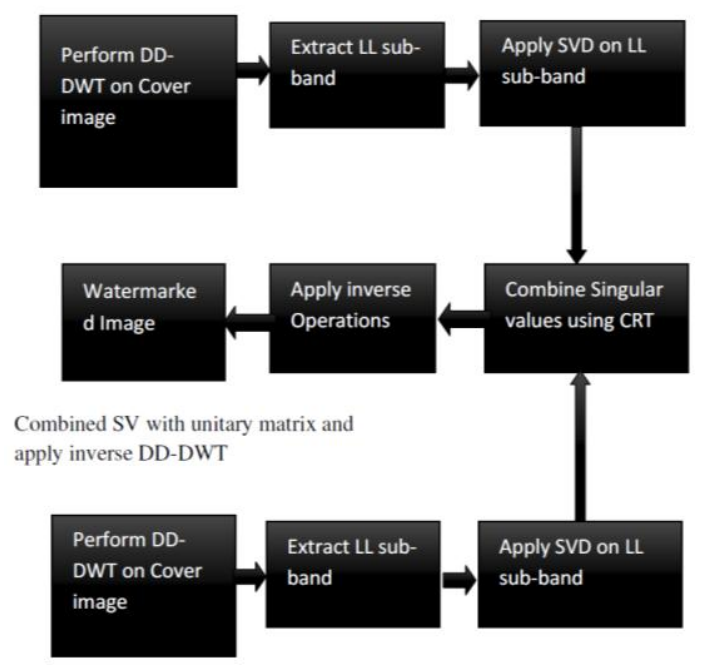

Figure 2. Block Diagram for Watermark Embedding Algorithm
Step 6: Finally, singular values are recovered and inverse DD-DWT is performed to produce the watermarked image.

\section{Watermark Extraction}

Step 1: For a given $\mathrm{N} \times \mathrm{N}$ watermarked image, apply the discrete wavelet Transform up to 3rd level, which produce a total of 9 bands of wavelet coefficients.

Step 2: The higher frequency sub-bands LL from 2nd level of wavelet decomposition are used to get 3rd level decomposition.

Step 3: Finally, the watermark is extracted from the selected wavelet coefficients by applying the singular value decomposition on the watermarked image and restoring it with the help of same CRT coefficients generated earlier.

Step 4: After extracting the final watermark, compares it with the original watermark, to find the any attacks happened in the original data.

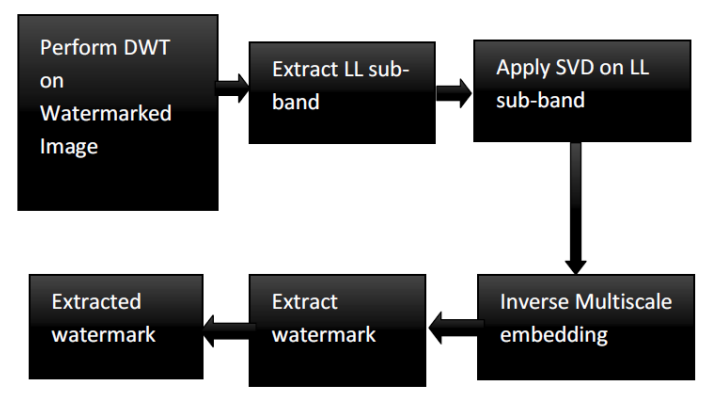

By removing singular values of cover image with respect to CRT coefficients

Figure 3. Block Diagram for Watermark Extraction Algorithm

\section{Results and Discussion}

The proposed DD-DWT and CRT based watermark technique has been applied to several images, including the sizes of Lena, Mandrill, and Barbara images. In these experiments, we have chosen a random sequence for creating the watermark matrices. The embedded watermarks cause imperceptible distortion at levels that provide reliable detection.

The channel parameters like Peak signal to Noise ratio (PSNR), Weighted Peak signal to Noise ratio (WPSNR), Mean signal Error (MSE) have been calculated.

The channel parameters have used to evaluate performance of both the systems. PSNR is the ratio between the maximum possible power of a signal and the power of corrupting noise that affects the fidelity of its representation. Because many signals have a very wide dynamic range, PSNR is usually 
expressed in terms of the logarithmic decibel $(\mathrm{dB})$ scale as:

$$
P S N R=10 \log _{10}\left(\frac{255^{2}}{E}\right) d B
$$

Where $\mathrm{E}$ is Mean Square Error, given by the mathematical expression as:

$$
E=\frac{1}{M \cdot N} \sum_{i=1}^{N} \sum_{j=1}^{M}\left[f(i, j)-f^{\prime}(i, j)\right]^{2}
$$

Where $f(i, j)$ is pixel value of original image of watermarked image and its logarithmic unit is $\mathrm{dB}$

The weighted PSNR (WPSNR) has been defined as an extension of the traditional PSNR. It weights each of the term of PSNR by local activity factor (linked to the local variance).The PSNR metric does not take into account image properties such as flat and textured regions. The watermark is embedding into textured regions and into edges, so the

PSNR is inadequate to measure image quality in this case. The solution of this problem is using weighted PSNR. NVF characterizes the local image properties and identifies texture and edge regions. This allows us to determine the optimal watermark location and strength for watermark embedding stage. The mathematical expression for WPSNR is given by:

$$
\text { WPSNR }=10\left(\mathrm{~L}_{\max }^{2}\right)(\mathrm{MSE} \times \mathrm{NVF})^{2}
$$

Where,

$$
L_{\max }=\frac{1}{\left(1+\theta \sigma x^{2}(I . J)\right)} \theta=D \sigma x_{\max }^{2}
$$

Which $\sigma \mathrm{x}_{\text {max }}^{2}$ is maximum local variance of a given image and $\mathrm{D} \in[50,150]$ is a determined parameter.

\subsection{Input Database}

The input database images shown in Figure 4 considered for the experimental purpose are Lena, Mandrill, and Barbara along with the single watermark (as shown in Figure 5). All images are in bmp format (bitmap image file).
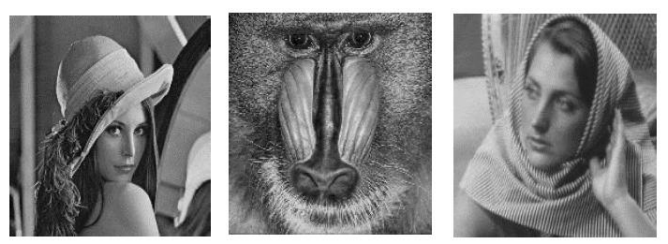

Figure 4. Input gray scale cover images considered during experiment (Lena, mandrill and Barbara)

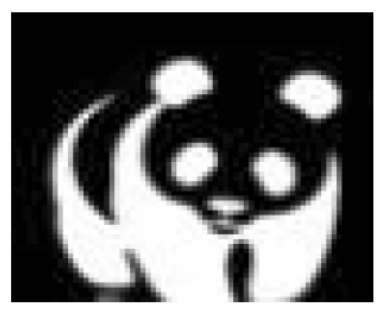

Figure 5. Input binary watermark image. All the experiments (with all three cover images) are done with this watermark

Table 1 shows the different type of wavelets using during the experiment. It is proved that higher level wavelets proved to be better as compare to traditional wavelets used earlier by some authors, different type of wavelet filters are applied using MATLAB's wavelet toolbox using function 'wfilters'.

\subsection{Comparative Analysis}

This section provide a comparison and validation of proposed methodology in terms of PSNR and MSE in order to prove the proposed CRT based DDDWT approach is better as compared to traditional DWT-CRT based approach.

Tables 2 and 3 in this section show the value of PSNR and MSE for the both methods in both the cases (test for robustness and test for invisibility). The wavelet used for comparison is traditional Haar wavelet (Sequence of rescaled "square-shaped" functions which together form a wavelet family or basis).

Figure 6 shows three watermarked images after applying the proposed embedding process using traditional Haar wavelet in CRT based DD-DWT model. The efficiency of proposed system in terms of invisibility is shown in tables of next sections. Figure 7 shows the input watermark image in proposed system and extracted watermark after embedding process. Haar wavelet is used and no channel noise is considered.

Table 1. Types of Wavelets Used in Proposed System

\begin{tabular}{|c|c|}
\hline $\begin{array}{c}\text { Serial } \\
\text { Number }\end{array}$ & Type of Wavelet \\
\hline 1 & 'db1' or Haar Wavelet \\
\hline 2 & 'db2' or Daubechies level two wavelets \\
\hline 3 & 'db4' or Daubechies level four wavelets \\
\hline 4 & 'sym2' or Symlets level two wavelets \\
\hline 5 & 'dmey' or Discrete Meyer Wavelets \\
\hline 6 & 'db45' or Daubechies level 45 wavelets \\
\hline
\end{tabular}



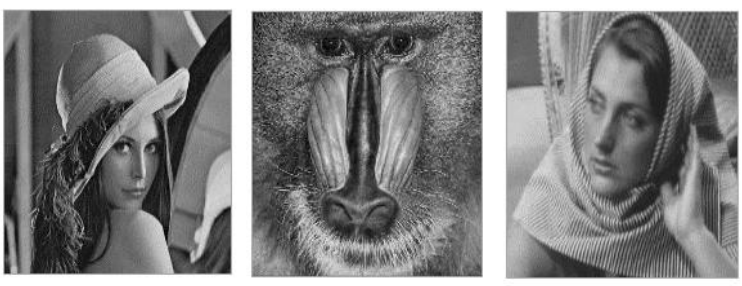

Figure 6. Watermarked Images using proposed system
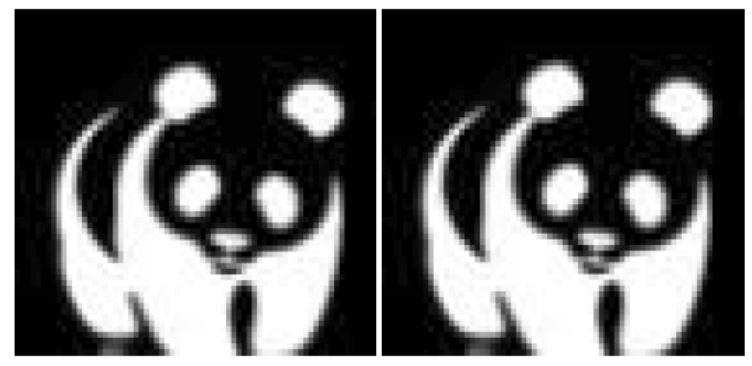

Figure 7. Input Watermark and Extracted watermark

Table 2 shows the comparison of Double Density CRT based system with DWT-CRT based approach in terms of PSNR and MSE for the case of robustness by comparing input and output watermark and Table 3 shows the comparison of proposed system with DWT-CRT based approach in terms of PSNR and MSE for the case of invisibility by comparing input cover image and watermarked image.

Figure 8 shows the comparison of proposed system with DWT-CRT in terms of PSNR (* for proposed approach and \# for traditional DWT-CRT, and comparing input and output watermark to check the robustness) and Fig. 9 shows the comparison of proposed system with DWT-CRT in terms of PSNR (* for proposed approach and \# for traditional DWTCRT) by comparing cover image and watermarked image to check the invisibility.

Table 2. Comparison of Proposed System with DWT-CRT Based Approach for Robustness

\begin{tabular}{|l|l|l|l|l|}
\hline \multirow{2}{*}{ Image Name } & \multicolumn{2}{|l|}{ Proposed System } & \multicolumn{2}{c|}{ DWT-CRT } \\
\cline { 2 - 5 } & PSNR & MSE & PSNR & MSE \\
\hline LENA & 56.6131 & 0.1418 & 36.0075 & 16.305 \\
\hline BARBARA & 54.2564 & 0.0512 & 40.5694 & 1.4951 \\
\hline MANDRILL & 53.9654 & 0.0400 & 38.5029 & 2.4062 \\
\hline
\end{tabular}

Table 3. Comparison of Proposed System with DWT-CRT Based Approach for Invisibility

\begin{tabular}{|l|l|l|l|l|}
\hline \multirow{2}{*}{ Image Name } & \multicolumn{2}{|l|}{$\begin{array}{l}\text { Proposed } \\
\text { System }\end{array}$} & \multicolumn{2}{c|}{ DWT-CRT } \\
\cline { 2 - 5 } & PSNR & MSE & PSNR & MSE \\
\hline LENA & 63.7896 & 0.0076 & 53.4684 & 0.2925 \\
\hline BARBARA & 64.5347 & 0.0022 & 49.6264 & 1.2894 \\
\hline MANDRILL & 61.9023 & 0.0041 & 47.9217 & 1.5697 \\
\hline
\end{tabular}

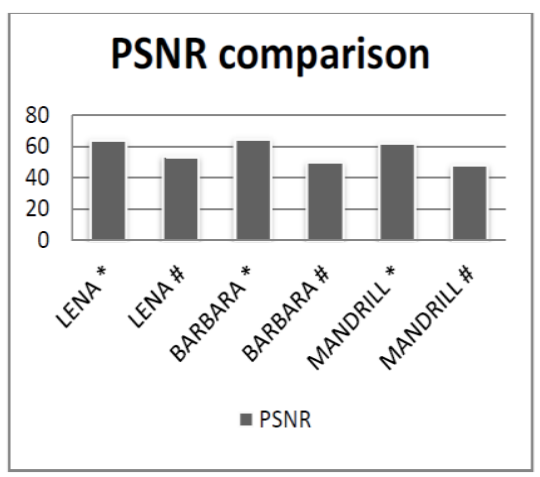

Figure 8. Comparative Study of two systems in terms of PSNR for Robustness

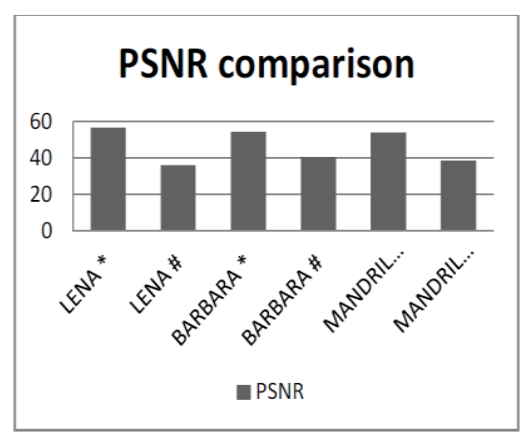

Figure 9. Comparative Study of two systems in terms of PSNR for Invisibility

Table 4 shows the efficiency of proposed system in terms of PSNR, MSE, WPSNR for different wavelet used for Lena image (*for robustness comparison between input and output watermark and \# for invisibility comparison between input cover image and watermarked image) and Table 5 shows the efficiency of proposed system in terms of PSNR, MSE, WPSNR and Correlation for different noise distortion used for Lena image. 
Table 4. Efficiency of Proposed System Using Different Wavelet

\begin{tabular}{|c|c|c|c|c|c|c|}
\hline Parameter & Haar & Db2 & Db4 & Sym & Mayer & DB45 \\
\hline PSNR $(*)$ & 63.7896 & 58.1665 & 64.162 & 58.1665 & 54.162 & 60.0261 \\
\hline MSE $\left(^{*}\right)$ & 0.02717 & 0.0099 & 0.0084 & 0.0099 & 0.0084 & 0.0064 \\
\hline $\begin{array}{c}\text { WPSNR } \\
(*)\end{array}$ & 67.114 & 74.2755 & 66.121 & 64.2755 & 66.121 & 63.0852 \\
\hline PSNR (\#) & 56.6131 & 62.8556 & 61.9118 & 62.8556 & 63.3780 & 63.7871 \\
\hline MSE (\#) & 0.14183 & 0.0336 & 0.0418 & 0.0336 & 0.02980 & 0.0271 \\
\hline
\end{tabular}

Table 5. Efficiency of Proposed System for Different Noise Distortion

\begin{tabular}{|c|c|c|c|c|c|c|}
\hline Parameters & Salt \& Pepper & Gaussian & Sharpened & Speckle & Rotation & Without \\
\hline PSNR & 51.2364 & 45.7369 & 68.2101 & 52.2922 & 76.1253 & 63.7896 \\
\hline MSE & 0.4891 & 1.7353 & 0.0098 & 0.3835 & 0.0015 & 0.02717 \\
\hline WPSNR & 77.1737 & 71.6507 & 94.7357 & 78.2528 & 101.394 & 67.114 \\
\hline CORR & 0.9721 & 0.9397 & 0.9696 & 0.9858 & 0.9598 & 0.99898 \\
\hline
\end{tabular}

Figure 10 shows the performance of different wavelets (haar, db2, db4, sym, meyer, db45) in proposed approach in terms of PSNR and WPSNR and Figure 11 shows the performance of proposed approach in presence of different noise factors in terms of PSNR and WPSNR.

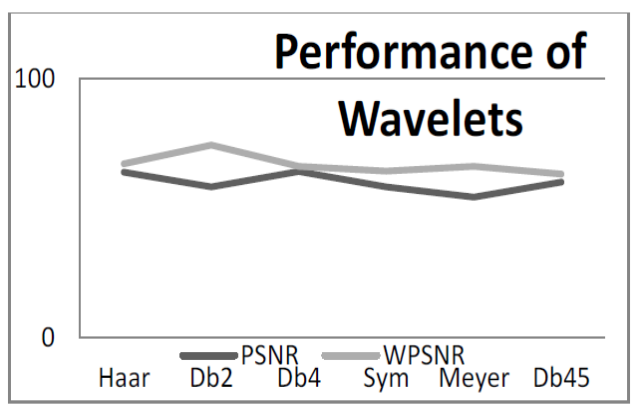

Figure 10. Performance of different wavelets

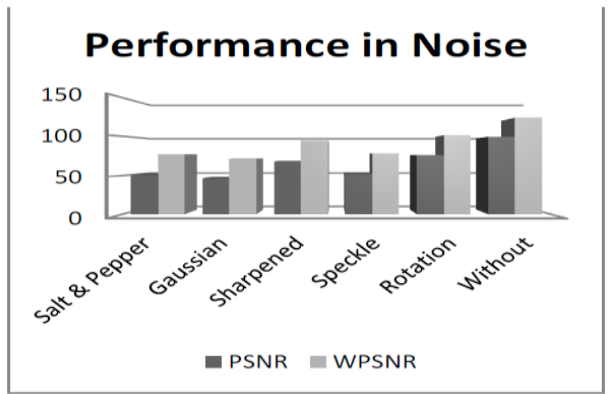

Figure 11. Performance of proposed system

\section{Conclusion}

The proposed CRT based DD-DWT technique provides better imperceptibility and higher robustness against attacks, as compared to traditional DWT and based scheme. Each watermark bit is embedded in various frequency bands and the information of the watermark bit is spread throughout large spatial regions. As a result, the watermarking technique is robust to attacks in both frequency and time domains.

The experimental results show the proposed embedding technique can survive the cropping of an image, image enhancement and the JPEG lossy compression. However, improvements in their performance can still be obtained by viewing the image watermarking problem as an optimization problem. In this paper we applied CRT model. By carefully using CRT scaling multiple watermarking and repeatedly embedding to harden the robustness are also possible. This technique could applied on colour images and also be applied to the multi resolution image structures with some modification about the choice of middle frequency coefficients. In this proposed method the values of the PSNRs of the watermarked images are always greater than $40 \mathrm{~dB}$ and it can effectively resist common image processing attacks, especially by JPEG compression and different kind of noises (Gaussian noise, speckle noise, salt and pepper noise, Poisson noise etc.). In proposed work, there's also an option for selecting a wavelet from different type of wavelets, experiments proved that Symmlet and Mexican hat wavelets proved better as compare to traditional wavelets like Haar and daubechies. A very good balance between robustness and imperceptibility has been achieved using this scheme as observers can evaluate the quality of the watermarked image as well as the recovered watermark to be good. Experimentation using various sizes of watermarks and different images enables a better understanding of the scheme. WPSNR is used to evaluate the perceptual quality of the watermarked image effectively and accurately.

\section{References}

[1] Hartung and M. Kutter, "Multimedia Watermarking Techniques,"Proc. of the IEEE, vol. 87, no. 7, pp. 10791107, July 1999.

[2] Chih-Chin Lai and Cheng-Chih Tsai.(November 2010) 'Digital Image Watermarking Using Discrete Wavelet Transform and Singular Value Decomposition',IEEE Transactions On Instrumentation And Measurement Vol.59, No.11, pp. 3060-3063.

[3] Chun-Shien Lu and Hong-Yuan Mark Liao. (October 2001). 'Multipurpose Watermarking for Image Authentication and Protection' IEEE Transactions On Image Processing Vol. 10, No.10, pp.1579- 1592. 
[4] V. Potdar, et al. "A Survey of Digital Image Watermarking Techniques", Proc. of the EEE International Conference on Industrial Informatics, Australia Perth, pp. 709-716, 2005.

[5] Liu Quan, AI Qingsong. “A combination of DCT based and SVD based watermarking, ICSP proceedings of IEEE International conference on signal processing, pp. 873-876, 2004.

[6] Feng Liu, et al. "A Watermarking Algorithm for Digital Image Based on DCT and SVD"; Congress on Image and Signal Processing, 2008.

[7] Emad E. Abdallah, et al. "A robust block-based image watermarking scheme using fast Hadamard Transform and Singular Value Decomposition" Proceedings of The 18th International Conference on Pattern Recognition, pp:673 676, 2006.

[8] Tang Xianghong, Yang Lianjie, YueHengli, Yin Zhongke, "A Watermarking Algorithm Based on the SVD and Hadamard Transform", Proceedings of International Conference on Communications, Circuits and Systems, Volume 2, pp. 874-877, 27-30 May 2005.

[9] Ali Al-Haj, "A Hybrid Digital Image Watermarking Algorithm", 4th International Conference on Innovations in Information Technology. pp: 690 - 694 Nov. 2007.

[10] Liu Liang and Sun Qi "A new SVD-DWT composite watermarking", ICSP proceedings of IEEE International conference on signal processing, 2006.

[11] Jung-Chun Liu, Chu-Hsing Lin, and Li-ChingKuo. "A Robust full band image watermarking scheme" Proceedings on IEEE, 2006.

[12] Qiang Li et al., "Adaptive DWT-SVD Domain Image Watermarking Using Human Visual Model”, Proceedings of 9th international conference on advanced communication Technology, Volume 3, pp: 1947 - 1951, Feb.2007.

[13] Y-P. Lai and C-C. Chang, "Parallel computational algorithms for generalized Chinese Remainder Theorem", Computers and Electrical Engineering, vol. 29, pp. 801811, 2003.

[14] M-S. Hwang, W-G. Tzeng and W-P. Yang, "An access control scheme based on Chinese Remainder Theorem and time stamp concept", Computers and Security, vol. 15, No. I, pp. 73-81, 1996.

[15] H. Chen, "CRT-based high-speed parallel architecture for long BCH encoding", IEEE Trans. Circuits and Systems-II, vol. 56, no. 8, pp. 684-86, Aug. 2009.

[16] C-C. Chang, Y-P. Hsieh and C-H. Lin, "Sharing secrets in stego images with authentication", Pattern Recognition, vol. 41, pp. 3130-3137, 2008.

[17] J. S. Shyong and Y-R. Chen, "Threshold secret image sharing by Chinese Remainder Theorem", Proc. IEEE Asia Pacific Services Computing Conference, Yilin, Taiwan, Dec. 2008, pp.1332-1337.
[18] J. C. Patra, A. Karthik and C. Bornand, "A Novel CRT-based watermarking technique for authentication of multimedia contents", Digital Signal Processing, vol. 20, no. 2, pp. 442-453, July 2009.

[19] J. C. Patra, J. E.Phua and D. Rajan, "DCT Domain watermarking scheme using Chinese Remainder Theorem for image authentication", Proc. IEEE Intl. Conf. Multimedia and Expo (ICME 2010), Singapore, July 2010, pp. 111-116.

[20] J. C. Patra, J. E. Phua and C. Bornand, "A novel DCT domain CRT based watermarking scheme for image authentication surviving JPEG compression", Digital Signal Processing, 20(6), 1597-1611, 2010. 